\title{
THE ALLEN TELESCOPE ARRAY Pi GHz SKY SURVEY. I. SURVEY DESCRIPTION AND STATIC CATALOG RESULTS FOR THE BOÖTES FIELD
}

\author{
Geoffrey C. Bower ${ }^{1}$, Steve Croft ${ }^{1}$, Garrett Keating ${ }^{1}$, David Whysong ${ }^{1}$, Rob Ackermann ${ }^{2}$, Shannon Atkinson $^{2}$, \\ Don Backer ${ }^{1}$, Peter Backus ${ }^{2}$, Billy Barott ${ }^{3}$, Amber Bauermeister ${ }^{1}$, Leo Blitz ${ }^{1}$, Douglas Bock ${ }^{1}$, Tucker Bradford ${ }^{2}$, \\ Calvin Cheng ${ }^{1}$, Chris Cork ${ }^{4}$, Mike Davis ${ }^{2}$, Dave DeBoer ${ }^{5}$, Matt DeXter ${ }^{1}$, John Dreher ${ }^{2}$, Greg Engargiola $^{1}$, Ed Fields ${ }^{1}$, \\ Matt Fleming $^{4}$, R. James Forster ${ }^{1}$, Colby Gutierrez-Kraybill ${ }^{1}$, G. R. Harp ${ }^{2}$, Carl Heiles ${ }^{1}$, Tamara Helfer $^{1}$, \\ Chat Hull ${ }^{1}$, Jane Jordan ${ }^{2}$, Susanne Jorgensen ${ }^{1}$, Tom Kilsdonk ${ }^{2}$, Casey Law ${ }^{1}$, Joeri Van Leeuwen ${ }^{6}$, John Lugten ${ }^{7}$, \\ Dave MacMahon ${ }^{1}$, Peter McMahon $^{8}$, Oren Milgrome ${ }^{1}$, Tom Pierson ${ }^{2}$, Karen Randall $^{2}$, John Ross $^{2}$, Seth Shostak $^{2}$, \\ Andrew Siemion $^{1}$, Ken SMOleK $^{2}$, Jill Tarter ${ }^{2}$, Douglas Thornton ${ }^{1}$, Lynn Urry ${ }^{1}$, Artyom Vitouchkine ${ }^{4}$, \\ Niklas Wadefalk ${ }^{9}$, Sandy Weinreb $^{10}$, Jack Welch $^{1}$, Dan Werthimer ${ }^{1}$, Peter K. G. Williams ${ }^{1}$, and Melvyn Wright ${ }^{1}$ \\ ${ }^{1}$ University of California, Berkeley, 601 Campbell Hall 3411, Berkeley, CA 94720, USA; gbower@astro.berkeley.edu \\ 2 SETI Institute, Mountain View, CA 94043, USA \\ ${ }^{3}$ Electrical and Systems Engineering Department, Embry-Riddle Aeronautical University, Daytona Beach, FL 32114, USA \\ ${ }^{4}$ Minex Engineering, Antioch, CA 94509, USA \\ ${ }^{5}$ CSIRO/ATNF, Epping, NSW 1710, Australia \\ ${ }^{6}$ ASTRON, NL-7990 AA Dwingeloo, The Netherlands \\ ${ }^{7}$ Lawrence Livermore National Laboratory, Livermore, CA 94550, USA \\ ${ }^{8}$ Electrical Engineering Department, Stanford University, Stanford, CA 94305, USA \\ ${ }^{9}$ Department of Microtechnology and Nanoscience-MC2, Chalmers University of Technology, SE-412 96 Göteborg, Sweden \\ ${ }^{10}$ California Institute of Technology, Department of Electrical Engineering, Pasadena, CA 91125, USA \\ Received 2010 July 9; accepted 2010 September 21; published 2010 December 2
}

\begin{abstract}
The Pi GHz Sky Survey (PiGSS) is a key project of the Allen Telescope Array. PiGSS is a $3.1 \mathrm{GHz}$ survey of radio continuum emission in the extragalactic sky with an emphasis on synoptic observations that measure the static and time-variable properties of the sky. During the 2.5 year campaign, PiGSS will twice observe $\sim 250,000$ radio sources in the $10,000 \mathrm{deg}^{2}$ region of the sky with $b>30^{\circ}$ to an rms sensitivity of $\sim 1 \mathrm{mJy}$. Additionally, sub-regions of the sky will be observed multiple times to characterize variability on timescales of days to years. We present here observations of a $10 \mathrm{deg}^{2}$ region in the Boötes constellation overlapping the NOAO Deep Wide Field Survey field. The PiGSS image was constructed from 75 daily observations distributed over a 4 month period and has an rms flux density between 200 and $250 \mu \mathrm{Jy}$. This represents a deeper image by a factor of 4-8 than we will achieve over the entire $10,000 \mathrm{deg}^{2}$. We provide flux densities, source sizes, and spectral indices for the 425 sources detected in the image. We identify $\sim 100$ new flat-spectrum radio sources; we project that when completed PiGSS will identify $10^{4}$ flat-spectrum sources. We identify one source that is a possible transient radio source. This survey provides new limits on faint radio transients and variables with characteristic durations of months.
\end{abstract}

Key words: radio continuum: galaxies - radio continuum: general - radio continuum: stars - surveys

Online-only material: color figures, machine-readable tables

\section{INTRODUCTION}

Synoptic surveys are increasingly important probes of the sky at all wavelengths. These surveys provide the opportunity to simultaneously probe the static and variable components of the universe through multiple observations of the sky. Complete static catalogs such as that of the NRAO VLA Sky Survey (Condon et al. 1998), the VLA FIRST Survey (Becker et al. 1995), the Sloan Digital Sky Survey (SDSS; Abazajian et al. 2003), and the ROSAT All Sky Survey (Voges et al. 1999) are essential to creating a complete picture of the astrophysics of a wide range of objects. These extant surveys predominantly emphasized a single static image of the sky. The next generation of large-scale surveys at all wavelengths has variability and the time domain as essential aspects. These surveys include at optical wavelengths the SDSS stripe 82 survey (Sesar et al. 2007), the Palomar Transient Factory (Rau et al. 2009; Law et al. 2009), Pan-STARRS, the LSST surveys, and at gamma-ray wavelengths the Fermi LAT catalog (Abdo et al. 2010).
At radio wavelengths, time domain surveys have a venerable history including the discovery of pulsars (Hewish et al. 1969) but are undergoing a significant renaissance. In particular, single dish surveys with sensitivity to short-timescale transients $(\lesssim 1 \mathrm{~s})$ have uncovered a wide range of neutron star phenomena, including single pulses detected from rotating radio transients (McLaughlin et al. 2006). These discoveries have spawned several new surveys (Siemion et al. 2010). Future versions of single-pulse surveys are proposed for several next-generation instruments such as ASKAP, LOFAR, MEERKAT, and the Square Kilometer Array (Macquart et al. 2010; Hessels et al. 2009). The enormous data volumes of these surveys require novel and dedicated signal processing techniques.

Imaging synoptic surveys, however, are in their infancy. They have been conducted systematically over limited area on the sky or assembled from archival data. Nevertheless, these limited surveys indicate that there are many phenomena to explore. VLA surveys of the Galactic center at low frequency, for example, have uncovered several transients without clear identification (Hyman et al. 2002, 2005, 2009). A comparison of the FIRST 
and NVSS surveys also uncovered a radio supernova and a radio transient without an optical counterpart (Levinson et al. 2002; Gal-Yam et al. 2006; Ofek et al. 2010). Bower et al. (2007) discovered 10 radio transients without counterparts in deep optical and radio images from nearly 1000 observations of a single field obtained weekly over 20 years. Recently, Becker et al. (2010) discovered a number of variable radio sources in observations of the Galactic plane. In addition to objects and events discovered through systematic searches, radio transients and variables have frequently been found through serendipity. Brunthaler et al. (2009), for instance, discovered a radio supernova (RSN) in M82 during VLBI observations intended to characterize the proper motion of the galaxy. The timescale of days to years includes known radio variability from radio supernovae and gamma-ray burst afterglows (Weiler et al. 2002), interstellar propagation such as extreme-scattering events (ESEs; Fiedler et al. 1987; Senkbeil et al. 2008), intrinsic active galactic nucleus (AGN) processes (Hughes et al. 1992), and compact-object binaries and stars (e.g., Berger et al. 2001; Bower et al. 2005; Osten et al. 2006).

de Zotti et al. (2010) summarize radio continuum surveys and their astrophysical uses. The NVSS (Condon et al. 1998) and the FIRST survey (Becker et al. 1995) constitute the best existing $1.4 \mathrm{GHz}$ radio surveys of the sky. The best existing high-frequency survey is the GB6 catalog, which has a detection threshold of $\sim 25$ mJy (Gregory et al. 1996). Large-area, highfrequency surveys that can provide statistical information about spectral indices and sources populations include the AT20G survey, which covers the southern hemisphere to a flux density threshold of $100 \mathrm{mJy}$ ( $91 \%$ completeness) at $20 \mathrm{GHz}$ (Murphy et al. 2010), and the $9 \mathrm{C}$ survey, which covers $520 \mathrm{deg}^{2}$ to $25 \mathrm{mJy}$ at $15 \mathrm{GHz}$ (Waldram et al. 2010).

The static component of large-scale surveys is valuable for a wide range of astrophysical problems. New radio surveys have the capability of providing spectral index information for radio sources, which can be critical for separating source classes. This includes the separation of star-forming galaxies from AGNs (Ivezić et al. 2002), identification of GHz-spectrumpeaked sources (Stanghellini et al. 1998), and identification of flat-spectrum radio sources. In particular, flat-spectrum catalogs are used for identification of blazars that may be gamma-ray counterparts (Healey et al. 2008; Massaro et al. 2009) and that may be useful for flux monitoring in determination of gravitational lensing constraints on cosmological parameters (Myers et al. 2003; Fassnacht et al. 2002).

We describe here a new synoptic survey at radio wavelengths conducted with the Allen Telescope Array (ATA) and provide initial results. The Pi GHz Sky Survey (PiGSS) is a key project of the ATA. Key goals are (1) to conduct a large-area survey of radio continuum at a frequency of $3.1 \mathrm{GHz}$ that approaches an order of magnitude more sensitivity than the best existing catalog at frequencies higher than $1.4 \mathrm{GHz}$ and (2) to explore the variable and transient components of the radio sky with a method that is unbiased by optical or high-energy observations. The first goal will produce spectral indices for an order of magnitude more sources than currently exist, enabling a wide range of science. This survey builds on a preliminary ATA project, the ATA Twenty Centimeter Survey (ATATS; Croft et al. 2010), which also demonstrates imaging quality and array performance. We describe the ATA, the survey, and its goals in Section 2 and the data reduction techniques in Section 3. We present our results in Section 4 and discuss these in Section 5. We summarize in Section 6.

\section{SURVEY DESCRIPTION}

The ATA is a new radio telescope designed to conduct surveys (Welch et al. 2009). The ATA is a pioneer of the large$\mathrm{N}$-small-diameter (LNSD) array design, which characterizes the international approach to the Square Kilometer Array design (Schilizzi et al. 2007). The survey speed is the rate per unit time at which a telescope covers solid angle to a fixed sensitivity. For a radio interferometer with $N$ elements of diameter $D$, the survey speed scales as $(N D)^{2}$, whereas the time to observe a single field scales proportional to the square of the total area $\left(N D^{2}\right)^{2}$. Thus, for a fixed collecting area, a smaller diameter $D$ will provide a faster survey speed. Unique features of the ATA are a wide field of view (2.5 at $1.4 \mathrm{GHz})$, compact configuration $\left(b_{\max }=300 \mathrm{~m}\right)$, a broadband feed that delivers the entire radio frequency band of $0.5-11 \mathrm{GHz}$ in orthogonal linear polarizations to the laboratory, and flexible digital signal processing that includes two correlators and three phased-array beamformers and uses four individual frequency tunings. Each correlator has a bandwidth of $104 \mathrm{MHz}$ in 1024 channels and provides full Stokes information. Williams \& Bower (2010) have exploited the broad frequency coverage of the telescope to measure continuous spectra of flux calibration standard sources and nearby star-forming galaxies, including M82, NGC 253, and Arp 220. The ATA was dedicated in Fall 2007; commissioning observations have been carried out over the past 2.5 years as array performance has improved.

The observing frequency was selected by balancing several factors: the need for higher frequency to achieve sufficient leverage to measure spectral indices; the expectation of greater amplitude and more rapid timescale variability from synchrotron sources at higher frequencies; the decreasing field of view and, hence, survey speed with increasing frequency; the improving sensitivity of the ATA at lower frequencies; and the absence of significant radio frequency interference (RFI). Two adjacent frequency bands were selected for the two correlators with center frequencies of 3.04 and $3.14 \mathrm{GHz}$.

Field selection for PiGSS was based on the goal of covering a very large area of extragalactic sky and overlapping with existing deep and wide area surveys. An overview of fields and specifications is given in Table 1. The North Galactic Cap at $b>30^{\circ}$ has been extensively imaged, in particular by the SDSS (Abazajian et al. 2003) at optical wavelengths and by the NVSS and FIRST surveys at a frequency of $1.4 \mathrm{GHz}$. Individual subfields were selected for similar reasons. The NOAO Deep Wide Field Survey (also known as the Boötes field; Jannuzi et al. 2004; Ashby et al. 2009) has been extensively imaged from radio to $\mathrm{X}$-ray wavelengths to significant depth and over an area as large as $10 \mathrm{deg}^{2}$. PiGSS is also targeting a $10 \mathrm{deg}^{2}$ region covering the Lockman hole for similar reasons. Both fields are seven-pointing hexagonally close-packed mosaics with a spacing between pointings equal to the voltage full width at half-maximum (FWHM), $\Delta \theta=0.78$ at $3.14 \mathrm{GHz}$. Additional $10 \mathrm{deg}^{2}$ fields will be selected and observed throughout the survey. A $250 \mathrm{deg}^{2}$ field overlapping the Boötes field was also observed and will be the subject of a subsequent paper.

The tiling of the North Galactic Pole (NGP) mosaic attempts to achieve a hexagonally close-packed spacing locally while minimizing the total number of pointings. Rows of pointings at constant declination are arranged with optimal spacing in right ascension for the minimum declination within that band of rows; i.e., $\Delta \alpha_{i}=\Delta \theta / \cos \left(\delta_{i, \min }\right)$, where $\delta_{i, \min }$ is the minimum declination within a band of rows. Pointing centers in adjacent 
Table 1

PiGSS Fields and Specifications

\begin{tabular}{|c|c|c|c|c|}
\hline Region Surveyed & North Galactic Cap & Boötes Field & Boötes Field & Lockman Hole \\
\hline Field area & $\begin{array}{c}10,000 \mathrm{deg}^{2} \\
b>30^{\circ}\end{array}$ & $\begin{array}{c}10 \mathrm{deg}^{2} \\
\alpha_{\text {cen }}=14^{\mathrm{h}} 32^{\mathrm{m}} \\
\delta_{\text {cen }}=34^{\circ} 16^{\prime}\end{array}$ & $\begin{array}{c}250 \mathrm{deg}^{2} \\
\alpha_{\text {cen }}=14^{\mathrm{h}} 32^{\mathrm{m}} \\
\delta_{\text {cen }}=34^{\circ} 16^{\prime}\end{array}$ & $\begin{array}{c}10 \mathrm{deg}^{2} \\
\alpha_{\text {cen }}=10^{\mathrm{h}} 49^{\mathrm{m}} \\
\delta_{\text {cen }}=58^{\circ} 20^{\prime}\end{array}$ \\
\hline Number of observations & 2 & 100 & 2 & 100 \\
\hline Cadence & 1 year & 1 day & 90 days & 1 day \\
\hline Time per pointing & 2 minutes & 2 minutes & 2 minutes & 2 minutes \\
\hline Detection threshold per epoch & $5 \mathrm{mJy}$ & $5 \mathrm{mJy}$ & $5 \mathrm{mJy}$ & $5 \mathrm{mJy}$ \\
\hline Final detection threshold & $4 \mathrm{mJy}$ & $1 \mathrm{mJy}$ & $4 \mathrm{mJy}$ & $1 \mathrm{mJy}$ \\
\hline Pointing angular spacing & 0.78 & 0.78 & 0.78 & 0.78 \\
\hline Angular resolution & $100^{\prime \prime}$ & $100^{\prime \prime}$ & $100^{\prime \prime}$ & $100^{\prime \prime}$ \\
\hline Position accuracy at $10 \sigma$ & $\lesssim 10^{\prime \prime}$ & $\lesssim 10^{\prime \prime}$ & $\lesssim 10^{\prime \prime}$ & $\lesssim 10^{\prime \prime}$ \\
\hline
\end{tabular}

rows are offset in right ascension by half of the spacing to provide the close-packed spacing. The number of rows for a fixed $\Delta \alpha_{i}$ is set by the constraint that the number of pointings per row does not exceed $110 \%$ of the optimal number for that row. The total number of pointings for the NGP is 19,940.

The integration time was selected with the goal of ultimately achieving a $5 \sigma$ detection threshold of $5 \mathrm{mJy}$ for a single epoch and $4 \mathrm{mJy}$ for the completed large-area survey. Typical observations consist of a loop that involves two minute observations of each pointing in one of the $10 \mathrm{deg}^{2}$ fields and approximately 50 pointings in the NGP (or other large) field. These observations are repeated between three and six times in an observing session with a duration of between 6 and $18 \mathrm{hr}$. This repetition gives more uniform $(u, v)$ coverage over the fields as well as increasing sensitivity. As the number of antennas available to the correlator increases, the number of observations per field will be reduced without losing sensitivity and $(u, v)$ coverage. Commissioning of new antennas and of new correlator capacity has been proceeding along with this survey. The observations reported here rely on data from about 25 dual polarization antennas.

The longest baseline of the ATA currently is $\sim 300 \mathrm{~m}$, providing $2^{\prime} \times 1^{\prime}$ resolution at $3.1 \mathrm{GHz}$. This resolution is a good match to NVSS $\left(\sim 45^{\prime \prime}\right)$, enabling accurate spectral indices. Source positions will be accurate to $10^{\prime \prime}$ or better. Matching with known FIRST sources will permit 1" accuracy positions for the majority of sources.

Source confusion from integrated faint sources provides a limit on the ultimate sensitivity that a static survey can obtain. Transient and variable surveys can, of course, detect variable sources that are fainter than the confusion limit. Condon (1974) derived the standard equations for confusion noise due to faint background sources. The results depend solely on the telescope resolution and the differential number count of sources per unit solid angle:

$$
n(S) d S=k S^{-\gamma} d S,
$$

where $k$ is a normalization constant and $\gamma \approx 2$ is the power-law index. The confusion noise is

$$
\sigma_{c}=\left(\frac{q^{3-\gamma}}{3-\gamma}\right)^{\frac{1}{\gamma-1}}\left(k \Omega_{e}\right)^{\frac{1}{\gamma-1}} .
$$

$\Omega_{e}$ is the effective beam area

$$
\Omega_{e}=\frac{\pi}{4} \theta_{1} \theta_{2} /[(\gamma-1) \ln 2],
$$

where $\theta_{1}$ and $\theta_{2}$ are the major and minor FWHM axes of the telescope beam. $q$ is an arbitrary parameter of the integration which is typically set to 5 . The detection threshold of an array is $\sim 5$ times the confusion noise for a nonvariable source. For the ATA, the rms confusion at $1.4 \mathrm{GHz}$ and $5.0 \mathrm{GHz}$ are $325 \mu \mathrm{Jy}$ and $40 \mu \mathrm{Jy}$, respectively, based on source counts at those wavelengths. In the absence of detailed source counts at $3.1 \mathrm{GHz}$, we can interpolate between those results and estimate the rms confusion at $3.1 \mathrm{GHz}$ to be $\sim 150 \mu \mathrm{Jy}$.

Cadence for synoptic observations is set to satisfy scheduling constraints and provide sensitivity to transient and variable sources on timescales of days to months. For the 10,000 deg 2 field, observations will be performed yearly, providing two samples of the flux density. For the $10 \mathrm{deg}^{2}$ fields, a cadence with intervals of days to years will be obtained. Following Bower et al. (2007), the two-epoch rate for a transient survey with $N_{e}$ epochs that cover an area $A$ to sensitivity $S$ is

$$
R(>S)=\frac{N_{t}}{\left(N_{e}-1\right) A(>S)}
$$

where $N_{t}$ is the number of transients detected. Here, $A(>S)$ refers to the solid angle over which a source of flux density $S$ or greater can be detected. Where no transient is detected, the $2 \sigma$ limit is $N_{t} \approx 3$ (Gehrels 1986). Croft et al. (2010) provide an updated version of this two-epoch rate. This rate applies to transients with a timescale comparable to or longer than the duration of individual epochs. Thus, the optimal approach to maximize the sensitivity to transients is to repeatedly observe the same field. Comparison to a prior epoch (such as NVSS or GB6) can increase $N_{e}$ by 1 .

\section{OBSERVING AND DATA REDUCTION}

PiGSS observing began in 2009 May. We report here on observations between 2009 May 20 and 2009 September 27. A total of 96 observations of the Boötes field were obtained, of which 75 were included in the final result. Observations in early 2009 August were interrupted for a period of two weeks due to forest fires in the region; observations in 2009 September were scheduled less densely than earlier in the campaign. We show the image rms of epochs with good data in Figure 1.

\subsection{Calibration and Flagging}

3C 286 was used as the calibrator for the amplitude scale as well as for short-term variations in antenna amplitude and phase gains. Calibrator observations were obtained hourly. A flux density of $9.7 \mathrm{Jy}$ was used, following the model of Baars et al. (1977). Observations of the bright calibrator 3C 295 on 2009 September 26 indicate that the amplitude scale is accurate 


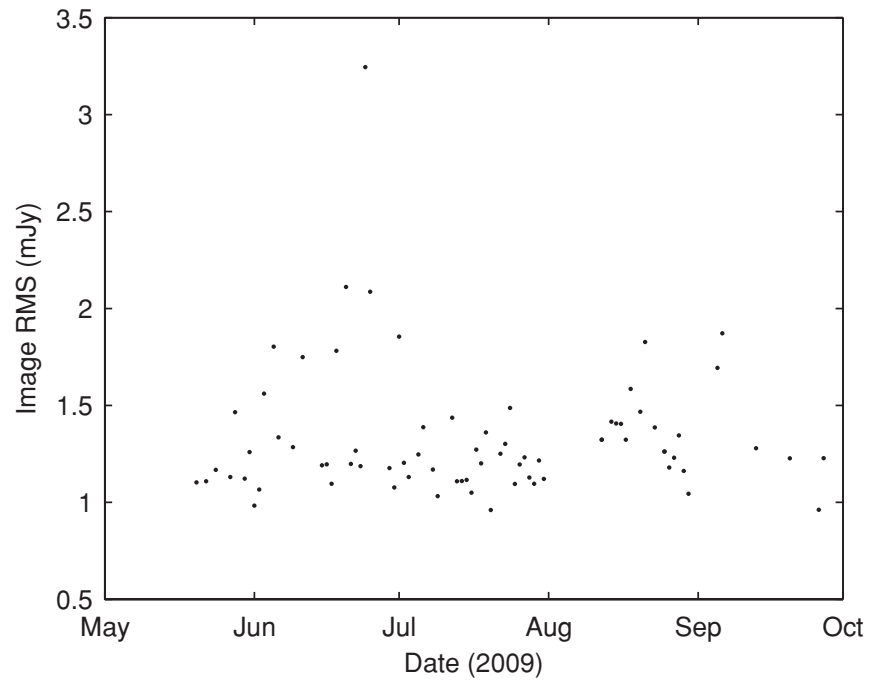

Figure 1. rms for each of the Boötes epochs as a function of time.

at the level of $\sim 1 \%$, consistent with earlier measurements by Williams \& Bower (2010) who find an accuracy of $~ 3 \%$. Total flux density in each of the two correlator bands was measured to be $10.84 \pm 0.02 \mathrm{Jy}$ and $10.55 \pm 0.05 \mathrm{Jy}$; the expected flux density at these frequencies is 10.845 and $10.493 \mathrm{Jy}$, respectively.

Data were reduced using the ARTIS package of scripts for RFI excision and amplitude calibration (Keating et al. 2009). ARTIS uses the MIRIAD package (Sault et al. 1995). Some additional RFI excision and rejection of bad antennas and baselines was performed by hand. The pipeline currently does not perform polarization leakage calibration; therefore, we do not provide polarimetric results in this paper. Accurate polarization calibration with the ATA has recently been demonstrated (Law et al. 2010); we anticipate that polarization calibration will be included in future versions of the pipeline. The lack of polarization calibration probably introduces a $\sim 1 \%$ error in total flux density and may limit dynamic range (Croft et al. 2010).

\subsection{Imaging}

All baselines shorter than $80 \lambda$ were rejected to exclude large-scale structure, solar interference, and cross-talk from closely spaced antennas from the resulting image. This has the effect of removing any sources with an angular size larger than $\sim 1^{\circ}$. Multi-frequency synthesis imaging was performed independently for each of the two frequency bands. A typical synthesized beam for PiGSS had a size of $120^{\prime \prime} \times 60^{\prime \prime}$ with a position angle that varied depending on the hour-angle range observed; we restored the image with a synthesized beam of $100^{\prime \prime} \times 100^{\prime \prime}$. A linear mosaic of all seven pointings at a single frequency was obtained using the primary-beam tapering algorithm described in Sault et al. (1996). The tapered map provides uniform noise across the image, which is useful for automated source identification, at the expense of uniform gain. We apply an after-the-fact amplitude calibration on sources by dividing fluxes by the tapering gain. The mosaics at the different frequencies were then combined with a weighted sum based on the measured noise in the maps. Finally, the mosaicked images were converted into the global sinusoid (GLS) geometry, which provides pixels of uniform area over the full field (Calabretta \& Greisen 2002). Without this correction, fluxes determined by our source detection algorithm have errors that increase with distance from the map center. We plot the rms of the final images from each epoch in Figure 1. The median rms for the set of images is $1.2 \mathrm{mJy}$.

The measured primary-beam FWHM of the ATA is $3.5 f^{-1}$, where $f$ is the frequency in $\mathrm{GHz}$ (Welch et al. 2009). We confirm through the examination of sources that are detected in two or more pointings that the best-fit primary beam is consistent with the nominal value of 1.13 for $3.1 \mathrm{GHz}$ (Hull et al. 2010). Mosaics are imaged to $5 \%$ of the beam sensitivity for a total area of $11.7 \mathrm{deg}^{2}$.

A deep image of the Boötes field was obtained by merging calibrated visibility data for each pointing for the entire observation period. This merged data set was imaged and then all pointings were combined to produce a linear mosaic of the field. This image (Figure 2) has an rms flux density between 216 and $250 \mu \mathrm{Jy}$, as measured in regions free of sources. The residual image after subtraction of CLEAN components has an rms of $200 \mu \mathrm{Jy}$. If we sum in quadrature the rms noise from each of the daily images, we obtain an expected rms noise of $145 \mu \mathrm{Jy}$. Additional noise in the image may be due to residual RFI, miscalibrated antennas, incomplete cleaning, and source confusion. The image includes data from approximately $150 \mathrm{hr}$ of observing using approximately half of the array. With the full array, the same image can be made in about $40 \mathrm{hr}$ of observing. The total area with sensitivity above $25 \%$ of the peak sensitivity $(\sim 1 \mathrm{mJy} \mathrm{rms})$ is $5.5 \mathrm{deg}^{2}$; above $50 \%$ of the peak sensitivity $\left(\sim 0.5 \mathrm{mJy} \mathrm{rms}\right.$ ) the total area is $3.3 \mathrm{deg}^{2}$ (Figure 3 ).

\subsection{Source Catalog Construction}

We constructed a catalog of sources using the MIRIAD program sfind (Hopkins et al. 2002). We constrain source fitting to be an elliptical Gaussian with a minimum size of the synthesized beam. This has the effect that many point sources will have an exact deconvolved size and position angle of 0 . We reject a small number of sources for which Gaussian fitting does not provide an adequate solution; these sources appear spurious (typically very elongated) when examined in the image domain. Source positions ( $\alpha, \delta$ in equinox J2000), flux densities, measured Gaussian sizes $\left(b_{\text {maj }}, b_{\text {min }}, \phi\right)$, and deconvolved Gaussian sizes $\left(b_{\text {maj }}^{\prime}, b_{\text {min }}^{\prime}, \phi^{\prime}\right)$ are tabulated in Table 2 . We determine the completeness of the catalog through comparison with the NVSS catalog, as discussed in the following section.

\section{BOÖTES FIELD RESULTS}

For the Boötes field, we apply a second cut to the catalog of a minimum flux density of $1 \mathrm{mJy}$, which is $4-5$ times the rms noise in the residual image. The total number of sources is then 425. The PiGSS catalog contains more than 10 times as many as sources as the GB6 catalog, which has 37 sources over this region. The minimum PiGSS flux is a factor of 2.5 and 18 fainter than the minimum NVSS and GB6 fluxes, respectively.

The Boötes field has been investigated systematically across the spectrum. Critical radio surveys against which to compare PiGSS data are the all sky NVSS and GB6 surveys and a deep Westerbork survey at $1400 \mathrm{MHz}$ (de Vries et al. 2002). We summarize the characteristics of these surveys in Table 3. We focus on comparison with NVSS and GB6 because these represent large-area surveys at resolutions similar to PiGSS. Comparison of the FIRST survey directly to PiGSS is likely to introduce source mismatches as sources are resolved into multiple components. Comparisons of FIRST and NVSS catalogs have been extensively investigated (e.g., Gal-Yam et al. 2006). 


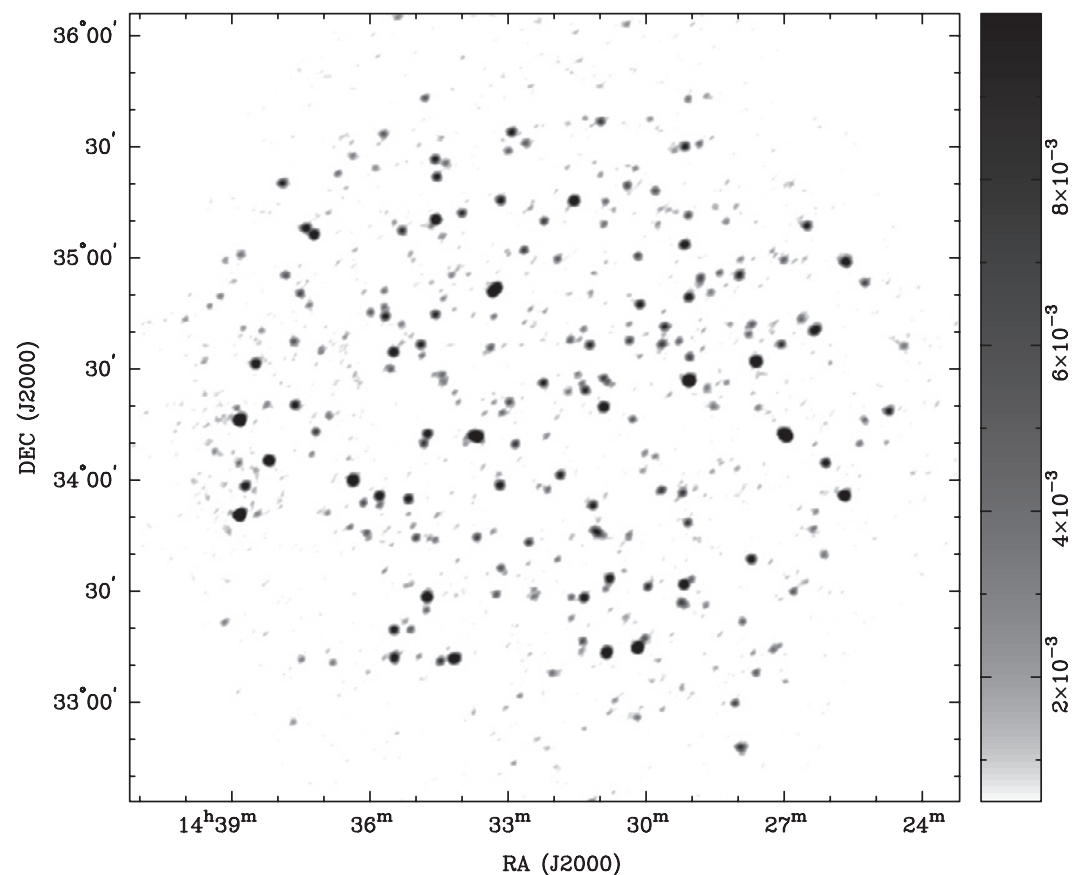

Figure 2. Image of the Boötes field at 3.1 GHz. The wedge demonstrates the flux range of the gray scale.

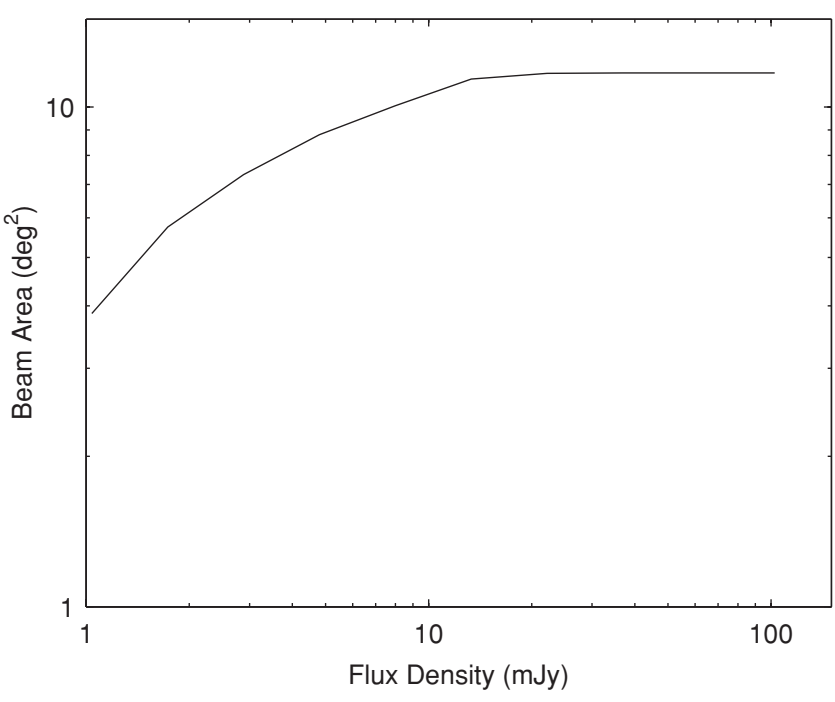

Figure 3. Imaged area as a function of flux density threshold.

We show images of individual sources and small regions in Figure 4. Catalog positions for sources from PiGSS and other surveys are indicated. For sources with simple structure, we see very good agreement between the PiGSS and NVSS catalog decomposition. The majority of PiGSS sources have this simple structure. In the case of more complex source regions such as Figures 4(c) and (d), the PiGSS decomposition tends to be simpler and produces a smaller number of total sources. The differences are due to the different resolutions, the more complete $(u, v)$ coverage of the PiGSS data, the different observing frequencies, and, possibly, different methods for complex source decomposition. GB6 sources are rare and the poorer angular resolution of GB6 leads to even greater blending of complex structures.

\subsection{Cross-identifications}

We match PiGSS sources with NVSS sources using a match radius $r_{m}=45^{\prime \prime}$. This radius is chosen to produce an expectation of $N_{\text {false }} \lesssim 1$ false match between PiGSS and NVSS, assuming a uniform distribution of sources. Clustering of sources will increase the number of false matches. However, a smaller match radius may lead to a mismatch between sources that are extended or resolved in NVSS. Approximately 80\% of PiGSS sources have a counterpart in NVSS; the remainder are likely to match to sources fainter than the NVSS flux limit (see the discussion below). We also match the PiGSS catalog to the GB6 catalog using $r_{m}=90^{\prime \prime}$, leading to $N_{\text {false }} \lesssim 1$. Only eight PiGSS sources have two NVSS sources within the PiGSS synthesized beam. Thus, no more than $2 \%$ of NVSS counterparts to PiGSS sources have their $1.4 \mathrm{GHz}$ fluxes underestimated by source-blending effects.

For the much deeper and higher angular resolution WSRT$1400, r_{m}=9^{\prime \prime}$ will produce $N_{\text {false }} \lesssim 1$. As discussed below, this match radius is comparable to the positional accuracy of our observations; use of a match radius of this size could lead to a substantial number of PiGSS sources not being identified. We select a match radius of $r_{m}=45^{\prime \prime}$ to accommodate the PiGSS positional uncertainty, leading to $N_{\text {false }} \sim 25$. Note that the minimum match radius is selected so the majority of PiGSS matches to WSRT-1400 are closer than this separation. Thirty-eight PiGSS sources $(\leqslant 10 \%)$ have multiple WSRT-1400 matches within the PiGSS synthesized beam. The characteristic error in the flux density for these multiple-match sources is an underestimate of the true $1.4 \mathrm{GHz}$ flux density by $16 \%$, if we assume that multiple-source decomposition does not create more accurate matches. The significant differences in source density and angular resolution between PiGSS and WSRT-1400 indicate that these matches are best used only when an NVSS or GB6 source is missing. We summarize the matches to all surveys and compute spectral indices $\left(S \propto v^{\alpha}\right)$ as a function of frequency for all of the matches in Table 4.

In Figure 5, we plot a histogram of the separation between the PiGSS and NVSS matched sources. Interior to the halfpower radius of the outer pointings, the median offset in the position is 8."3; outside that radius, the median offset increases to 13 ".7. There is some evidence for systematic shifts in position 
Table 2

PiGSS Sources

\begin{tabular}{|c|c|c|c|c|c|c|c|c|c|c|c|c|}
\hline Name & $\begin{array}{l}\text { R.A. } \\
\text { (hr) }\end{array}$ & $\begin{array}{l}\text { Decl. } \\
\text { (deg) }\end{array}$ & $\begin{array}{c}\Delta \alpha \\
(\operatorname{arcsec})\end{array}$ & $\begin{array}{c}\Delta \delta \\
(\operatorname{arcsec})\end{array}$ & $\begin{array}{c}b_{\text {maj }} \\
(\operatorname{arcsec})\end{array}$ & $\begin{array}{c}b_{\min } \\
(\operatorname{arcsec})\end{array}$ & $\begin{array}{c}\phi \\
(\mathrm{deg})\end{array}$ & $\begin{array}{c}b_{\text {maj }}^{\prime} \\
(\operatorname{arcsec})\end{array}$ & $\begin{array}{c}b_{\min }^{\prime} \\
(\operatorname{arcsec})\end{array}$ & $\begin{array}{c}\phi^{\prime} \\
(\mathrm{deg})\end{array}$ & $\begin{array}{c}\text { Flux } \\
(\mathrm{mJy})\end{array}$ & $\begin{array}{c}\text { Err } \\
(\mathrm{mJy})\end{array}$ \\
\hline $\mathrm{J} 142318+344210$ & 14.38826 & 34.70267 & 17.9 & 17.8 & 100.0 & 100.0 & 0.0 & 0.0 & 0.0 & 0.0 & 18.01 & 4.45 \\
\hline $\mathrm{J} 142402+344518$ & 14.40044 & 34.75503 & 11.4 & 11.3 & 150.4 & 122.6 & 55.0 & 112.3 & 70.9 & 88.7 & 16.49 & 2.59 \\
\hline $\mathrm{J} 142421+343857$ & 14.40570 & 34.64913 & 19.5 & 19.5 & 100.0 & 100.0 & 0.0 & 0.0 & 0.0 & 0.0 & 6.21 & 1.68 \\
\hline $\mathrm{J} 142426+343602$ & 14.40719 & 34.60060 & 3.5 & 3.5 & 136.8 & 117.5 & 65.8 & 93.4 & 61.7 & 9.9 & 30.33 & 1.47 \\
\hline $\mathrm{J} 142430+341914$ & 14.40831 & 34.32064 & 19.8 & 19.8 & 100.0 & 100.0 & 0.0 & 0.0 & 0.0 & 0.0 & 4.04 & 1.11 \\
\hline $\mathrm{J} 142440+343757$ & 14.41101 & 34.63258 & 9.8 & 9.5 & 185.4 & 103.9 & 63.9 & 156.1 & 28.2 & -61.2 & 10.00 & 1.32 \\
\hline $\mathrm{J} 142445+341832$ & 14.41253 & 34.30878 & 2.1 & 2.1 & 111.5 & 102.7 & -74.7 & 49.3 & 23.4 & -40.0 & 42.03 & 1.22 \\
\hline $\mathrm{J} 142447+345317$ & 14.41295 & 34.88818 & 15.9 & 15.9 & 100.0 & 100.0 & 0.0 & 0.0 & 0.0 & 0.0 & 8.54 & 1.88 \\
\hline $\mathrm{J} 142448+340957$ & 14.41339 & 34.16577 & 10.7 & 10.7 & 100.0 & 100.0 & 0.0 & 0.0 & 0.0 & 0.0 & 6.18 & 0.92 \\
\hline $\mathrm{J} 142458+342527$ & 14.41625 & 34.42421 & 10.3 & 10.3 & 164.4 & 105.3 & 66.5 & 130.5 & 33.0 & -30.2 & 5.89 & 0.84 \\
\hline $\mathrm{J} 142503+334405$ & 14.41761 & 33.73465 & 18.4 & 18.4 & 100.0 & 100.0 & 0.0 & 0.0 & 0.0 & 0.0 & 4.90 & 1.24 \\
\hline $\mathrm{J} 142516+345310$ & 14.42118 & 34.88600 & 2.5 & 2.5 & 117.6 & 105.3 & 88.9 & 61.9 & 33.0 & -53.6 & 38.86 & 1.34 \\
\hline $\mathrm{J} 142517+341606$ & 14.42129 & 34.26831 & 5.0 & 5.0 & 100.0 & 100.0 & 0.0 & 0.0 & 0.0 & 0.0 & 9.71 & 0.67 \\
\hline $\mathrm{J} 142523+340944$ & 14.42302 & 34.16212 & 5.3 & 5.3 & 100.0 & 100.0 & 0.0 & 0.0 & 0.0 & 0.0 & 8.83 & 0.64 \\
\hline $\mathrm{J} 142536+331215$ & 14.42679 & 33.20403 & 10.1 & 10.2 & 140.3 & 115.9 & -26.5 & 98.4 & 58.6 & 78.3 & 16.39 & 2.29 \\
\hline $\mathrm{J} 142541+345848$ & 14.42811 & 34.98005 & 1.7 & 1.7 & 130.0 & 110.2 & 67.6 & 83.1 & 46.3 & 86.8 & 105.85 & 2.45 \\
\hline $\mathrm{J} 142543+335544$ & 14.42853 & 33.92887 & 1.6 & 1.6 & 100.0 & 100.0 & 0.0 & 0.0 & 0.0 & 0.0 & 123.69 & 2.68 \\
\hline $\mathrm{J} 142558+351311$ & 14.43279 & 35.21974 & 18.8 & 18.8 & 100.0 & 100.0 & 0.0 & 0.0 & 0.0 & 0.0 & 5.43 & 1.41 \\
\hline $\mathrm{J} 142601+343129$ & 14.43357 & 34.52459 & 17.7 & 17.7 & 100.0 & 100.0 & 0.0 & 0.0 & 0.0 & 0.0 & 2.69 & 0.66 \\
\hline $\mathrm{J} 142607+340433$ & 14.43531 & 34.07572 & 1.6 & 1.6 & 100.0 & 100.0 & 0.0 & 0.0 & 0.0 & 0.0 & 27.45 & 0.61 \\
\hline
\end{tabular}

(This table is available in its entirety in a machine-readable form in the online journal. A portion is shown here for guidance regarding its form and content.)

Table 3

Boötes Surveys

\begin{tabular}{lcccc}
\hline \hline Survey & $\begin{array}{c}\text { Frequency } \\
(\mathrm{GHz})\end{array}$ & $\begin{array}{c}\text { Min. Flux } \\
(\mathrm{mJy})\end{array}$ & $\begin{array}{c}\text { Resolution } \\
(\operatorname{arcsec} \times \operatorname{arcsec})\end{array}$ & $\begin{array}{c}\text { FOV } \\
\left(\mathrm{deg}^{2}\right)\end{array}$ \\
\hline WSRT-1400 & 1.4 & 0.14 & $13 \times 27$ & 7.0 \\
NVSS & 1.4 & 2.5 & $45 \times 45$ & $3 \times 10^{4}$ \\
FIRST & 1.4 & 1.0 & $5 \times 5$ & $10^{4}$ \\
PiGSS & 3.1 & 0.8 & $100 \times 100$ & 11.7 \\
GB6 & 4.9 & 18 & $130 \times 130$ & $2 \times 10^{4}$ \\
\hline
\end{tabular}

at radii approaching two times the half-power radius (Figure 6). These shifts are not described as a simple linear shift or a rotation, either around the field center or around individual pointing centers. Tests with full three-dimensional imaging in CASA appear to indicate that these are not due to phase errors introduced by ignoring the $w$-term. Bandwidth and time smearing are not significant at these radii. These localized shifts may be due to primary-beam phase errors at radii beyond the half-power point. The accuracy of matching with WSRT-1400 sources is comparable to that achieved with NVSS; the median separation between PiGSS and WSRT-1400 sources is 10'.5.

We use multiple catalogs to identify PiGSS sources without associations (Figure 7). The absence of an NVSS counterpart is unsurprising for low flux density sources given the lower detection threshold for PiGSS; 89 PiGSS sources are unmatched to NVSS. If we match against the WSRT-1400 survey as well as NVSS, we are able to identify more sources, leaving us with 35 unidentified sources. Many of the remaining unmatched PiGSS sources are outside the boundary of the WSRT-1400 survey; i.e., these sources could only be matched to NVSS. The different resolutions and $(u, v)$ coverages of these surveys also lead to mismatches. For instance, we see extended structure associated with a bright source that does not appear in the NVSS image of the field (Figure 8). This resolved source is represented by the spike at $>8 \mathrm{mJy}$ in the histogram. If we further exclude all resolved sources from the histogram, we are left with sources with flux densities less than $2.5 \mathrm{mJy}$. Further excluding sources outside the half-power sensitivity, we find five sources with flux densities of $\sim 1.5 \mathrm{mJy}$ that have no match in any of the catalogs. We show images of these sources in Figure 9. Of these sources, one is adjacent to a very bright source with resolved structure and is possibly a sidelobe of that source or is missed in other surveys due to confusion with that bright source; three have probable WSRT-1400 matches that are beyond the $45^{\prime \prime}$ match radius. Only J143621+334120 has no apparent match and no reason to exclude it as a real source.

All NVSS sources with flux densities greater than $5 \mathrm{mJy}$ are matched to PiGSS sources either through a clear one-to-one match or with an association with a resolved source that has a different decomposition into components in PiGSS and NVSS. Fifty-three NVSS sources with flux densities between 2.5 and 5 mJy are not matched to PiGSS sources; this is primarily due to a combination of faint $3.1 \mathrm{GHz}$ emission that is not detected in PiGSS, source confusion, and a few sources that are missed by the PiGSS detection algorithm.

All but two GB6 sources have matches to PiGSS sources. GB6 source J143921+344803 falls in between two PiGSS sources with a separation of $\sim 4^{\prime}$ and is probably a blend of these two. GB6 source J142554+343552, which has a flux density of $21 \mathrm{mJy}$, has no apparent counterpart in PiGSS, NVSS, or WSRT1400 . The nearest PiGSS source is $\sim 5^{\prime}$ away. The field contains a number of nearby galaxies identified by SDSS but there are no AGN or known compact objects. There is no known reason to exclude this GB6 source as a transient source but without deeper access to the GB6 data we cannot determine whether it is real or spurious.

\subsection{Spectral Indices}

Spectral indices are tabulated in Table 4 and plotted in Figure 10. There is good agreement between spectral indices determined with NVSS and WSRT-1400 data. The median spectral indices with the two surveys are -0.68 and -0.65 , respectively. Excluding 25 sources that have very large spectral indices in one or the other survey (and are, therefore, likely to 

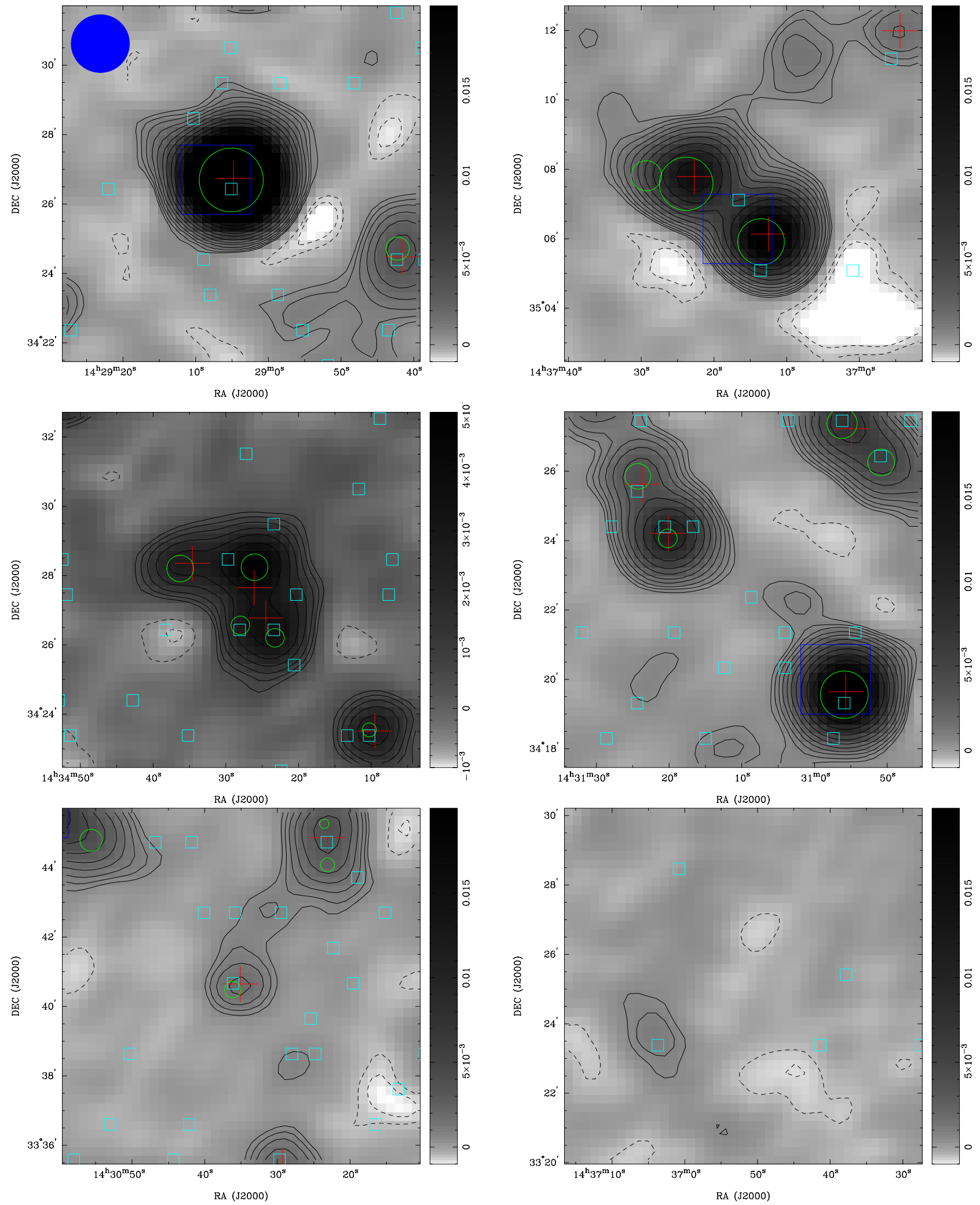

Figure 4. Images of regions within the large image: (a) one of the brightest sources in the field with a flux density of $113 \pm 1 \mathrm{mJy}$, (b) a double source, (c) a complex of faint sources, (d) another complex of sources, (e) the faintest source in the catalog, and (f) an empty field. All images have contours that are $-4,-2.8,2.8,4,5.6$, $8,11.2, \ldots, 256$ times $0.2 \mathrm{mJy}$. The synthesized beam is shown in (a). Crosses indicate PiGSS sources. Circles indicate NVSS sources, with area proportional to the logarithm of the flux density. Large, blue squares represent GB6 sources. Small cyan squares represent WSRT-1400 sources. Each image is $10^{\prime} \times 10^{\prime}$.

(A color version of this figure is available in the online journal.) 
Table 4

Radio Matches to PiGSS Sources

\begin{tabular}{|c|c|c|c|c|c|c|c|c|c|}
\hline Name & $\begin{array}{l}S_{1400} \\
(\mathrm{mJy})\end{array}$ & $\alpha_{1400}$ & $\Delta \alpha_{1400}$ & $\begin{array}{l}S_{\mathrm{NVSS}} \\
(\mathrm{mJy})\end{array}$ & $\alpha_{\mathrm{NVSS}}$ & $\Delta \alpha_{\mathrm{NVSS}}$ & $\begin{array}{l}S_{4850} \\
(\mathrm{mJy})\end{array}$ & $\alpha_{4850}$ & $\Delta \alpha_{4850}$ \\
\hline $\mathrm{J} 142318+344210$ & 0.00 & 0.00 & 0.00 & 34.30 & -0.81 & 0.31 & 0.00 & 0.00 & 0.00 \\
\hline $\mathrm{J} 142426+343602$ & 0.00 & 0.00 & 0.00 & 47.60 & -0.57 & 0.06 & 0.00 & 0.00 & 0.00 \\
\hline $\mathrm{J} 142440+343757$ & 0.00 & 0.00 & 0.00 & 15.90 & -0.59 & 0.17 & 0.00 & 0.00 & 0.00 \\
\hline $\mathrm{J} 142445+341832$ & 0.00 & 0.00 & 0.00 & 82.10 & -0.85 & 0.04 & 22.00 & -1.44 & -0.41 \\
\hline $\mathrm{J} 142447+345317$ & 0.00 & 0.00 & 0.00 & 20.70 & -1.12 & 0.28 & 0.00 & 0.00 & 0.00 \\
\hline $\mathrm{J} 142448+340957$ & 0.00 & 0.00 & 0.00 & 8.10 & -0.34 & 0.20 & 0.00 & 0.00 & 0.00 \\
\hline $\mathrm{J} 142516+345310$ & 0.00 & 0.00 & 0.00 & 65.90 & -0.67 & 0.04 & 28.00 & -0.73 & -0.33 \\
\hline $\mathrm{J} 142517+341606$ & 0.00 & 0.00 & 0.00 & 16.30 & -0.65 & 0.10 & 0.00 & 0.00 & 0.00 \\
\hline $\mathrm{J} 142523+340944$ & 0.00 & 0.00 & 0.00 & 20.60 & -1.07 & 0.10 & 0.00 & 0.00 & 0.00 \\
\hline $\mathrm{J} 142541+345848$ & 262.60 & -1.15 & 0.06 & 154.70 & -0.48 & 0.03 & 69.00 & -0.95 & -0.23 \\
\hline $\mathrm{J} 142543+335544$ & 322.78 & -1.21 & 0.06 & 303.10 & -1.13 & 0.03 & 79.00 & -0.99 & -0.23 \\
\hline $\mathrm{J} 142558+351311$ & 0.00 & 0.00 & 0.00 & 9.30 & -0.68 & 0.34 & 0.00 & 0.00 & 0.00 \\
\hline $\mathrm{J} 142601+343129$ & 5.43 & -0.89 & 0.31 & 3.60 & -0.37 & 0.36 & 0.00 & 0.00 & 0.00 \\
\hline $\mathrm{J} 142607+340433$ & 37.73 & -0.40 & 0.06 & 34.60 & -0.29 & 0.03 & 28.00 & 0.04 & -0.32 \\
\hline $\mathrm{J} 142609+333949$ & 29.78 & -0.89 & 0.09 & 27.80 & -0.81 & 0.08 & 0.00 & 0.00 & 0.00 \\
\hline $\mathrm{J} 142620+335127$ & 13.96 & -1.30 & 0.16 & 11.50 & -1.05 & 0.17 & 0.00 & 0.00 & 0.00 \\
\hline $\mathrm{J} 142621+344021$ & 87.01 & -0.74 & 0.05 & 38.60 & 0.29 & 0.02 & 36.00 & -0.66 & -0.31 \\
\hline $\mathrm{J} 142621+340937$ & 6.51 & -1.09 & 0.25 & 5.00 & -0.76 & 0.27 & 0.00 & 0.00 & 0.00 \\
\hline $\mathrm{J} 142623+334643$ & 4.51 & 1.28 & 0.09 & 2.60 & 1.98 & 0.25 & 0.00 & 0.00 & 0.00 \\
\hline $\mathrm{J} 142632+350831$ & 0.00 & 0.00 & 0.00 & 95.00 & -1.13 & 0.05 & 28.00 & -0.72 & -0.33 \\
\hline
\end{tabular}

Notes. Zeros indicate no match.

(This table is available in its entirety in a machine-readable form in the online journal. A portion is shown here for guidance regarding its form and content.)

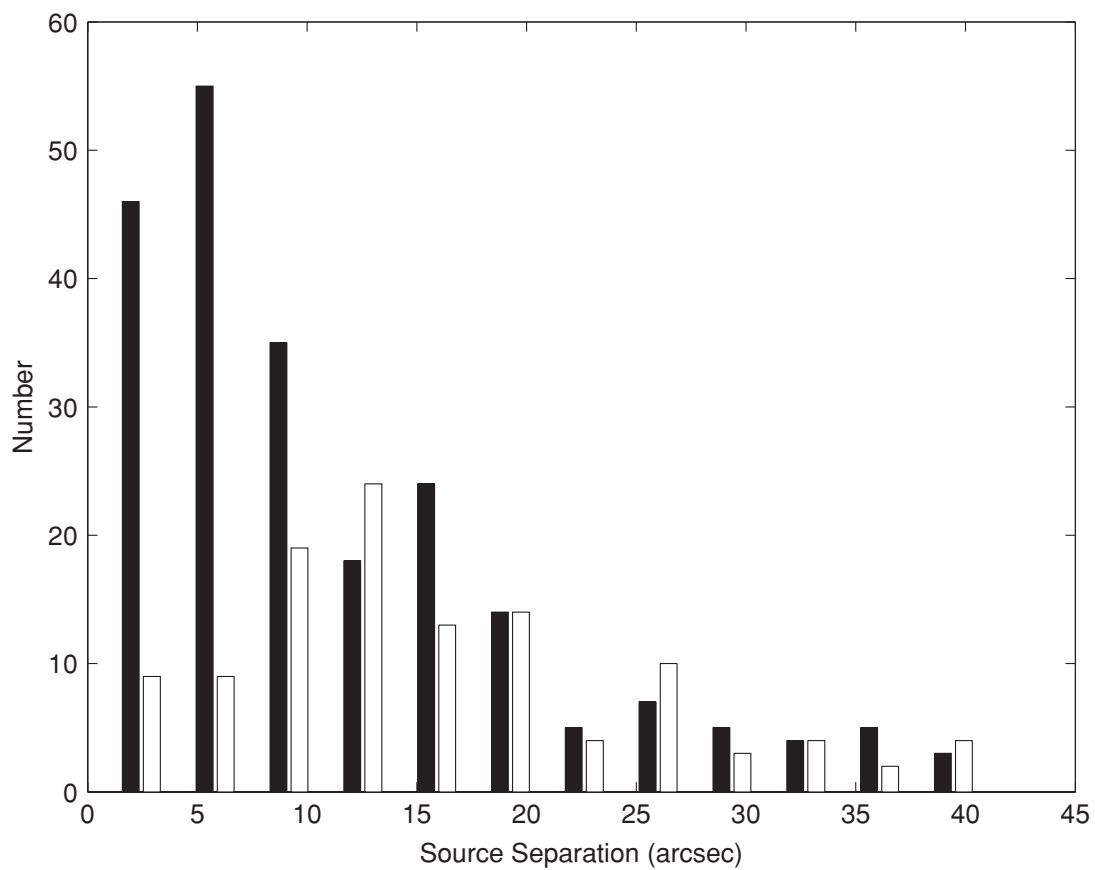

Figure 5. Histogram of separation between PiGSS and NVSS sources. Solid bars are for sources within the half-power radii of the outer pointings; open bars are for sources beyond that limit.

be mismatched), the rms difference in spectral index relative to NVSS and WSRT-1400 is 0.17. This is an upper limit on the systematic error in the NVSS spectral indices because it includes the relative calibration error between NVSS and WSRT-1400. The drop in median spectral index at low flux densities is a selection effect due to the $2.5 \mathrm{mJy}$ flux limit of the NVSS catalog; inverted or flat-spectrum PiGSS sources will not be detected in NVSS at low flux density. GB6 spectral indices have a larger scatter, caused by a larger error in the GB6 flux densities, the coarser angular resolution of GB6, and, possibly, higher variability among these sources. Only one GB6 source (out of 37) has a PiGSS source within 90 arcsec radius, which is well beyond the half-power radius of $70 \mathrm{arcsec}$. Thus, we are not significantly affected by source-blending effects in the PiGSS-GB6 spectral indices.

\subsection{Number Counts}

We construct number counts for the PiGSS, NVSS, and GB6 sources in the field (Figure 11). As expected, PiGSS number counts fall in between the NVSS and GB6 number counts since 


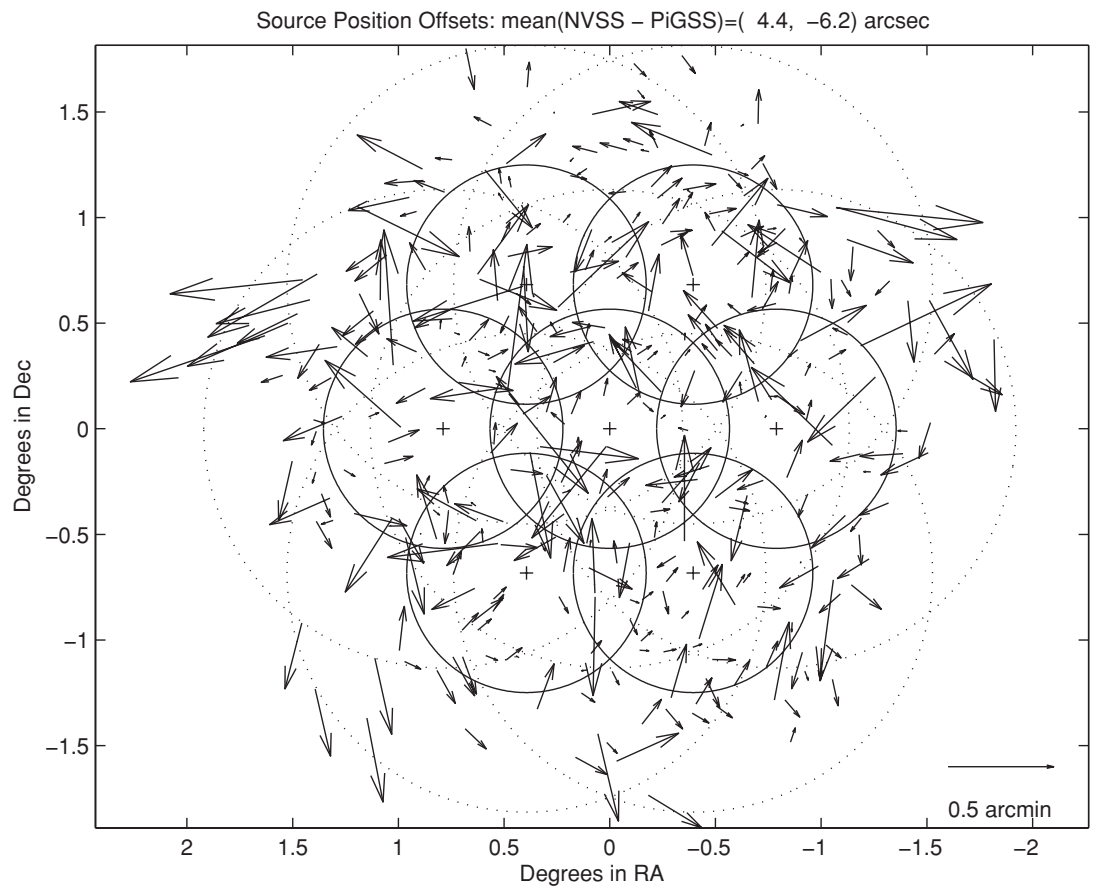

Figure 6. Vectors of position offsets between NVSS and PiGSS. The mean offset has been removed. A scale arrow in the lower right indicates 0.5 offset. Note that arrows are much larger than actual scale. Crosses indicate the centers of each pointing, solid circles indicate the half-power radii, and dashed circles indicate two times the half-power radii.

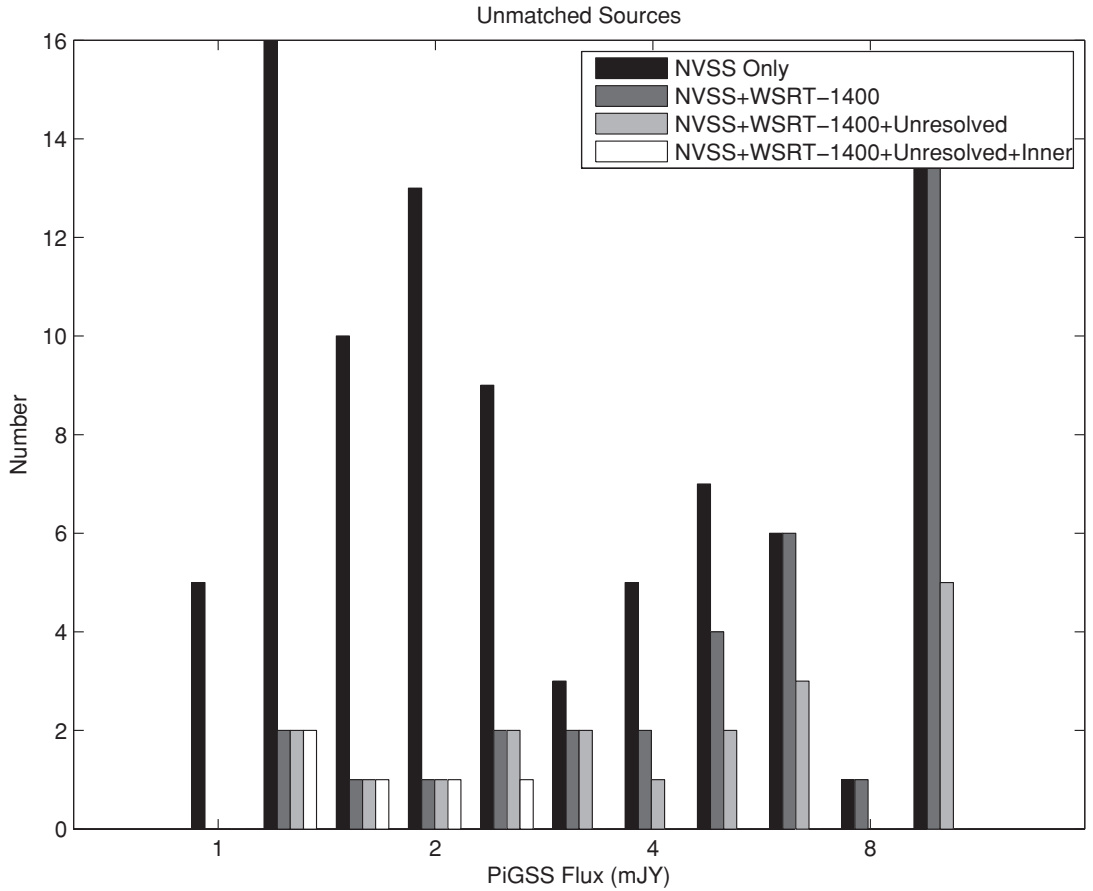

Figure 7. Histogram of PiGSS sources not identified in matches with other catalogs. See the text for details.

the average spectral index is $\sim-0.7$. This result indicates that we are detecting the expected population in this field.

\section{DISCUSSION}

\subsection{Variable and Transient Sources}

A major goal of PiGSS is to identify and characterize variable and transient radio sources. The results of this paper, specifically the catalog of sources in comparison with existing radio catalogs of the richly surveyed Boötes field, enable us to investigate variability with a characteristic timescale of $\sim 4$ months relative to earlier snapshot epochs. That is, transient sources that appear in the PiGSS data are most likely to have a lifetime comparable to the total integration time of this survey; variable sources can have evolved even more slowly, i.e., over the course of years from prior epochs to the present. Brighter, shorter-duration transients are possible but less probable if they have source populations that increase with decreasing flux density. The reverse analysis-identification of sources present in NVSS, WSRT-1400, or other surveys but not present in PiGSS-provides an opportunity to probe source populations with characteristic timescales that match the shorter snapshot 


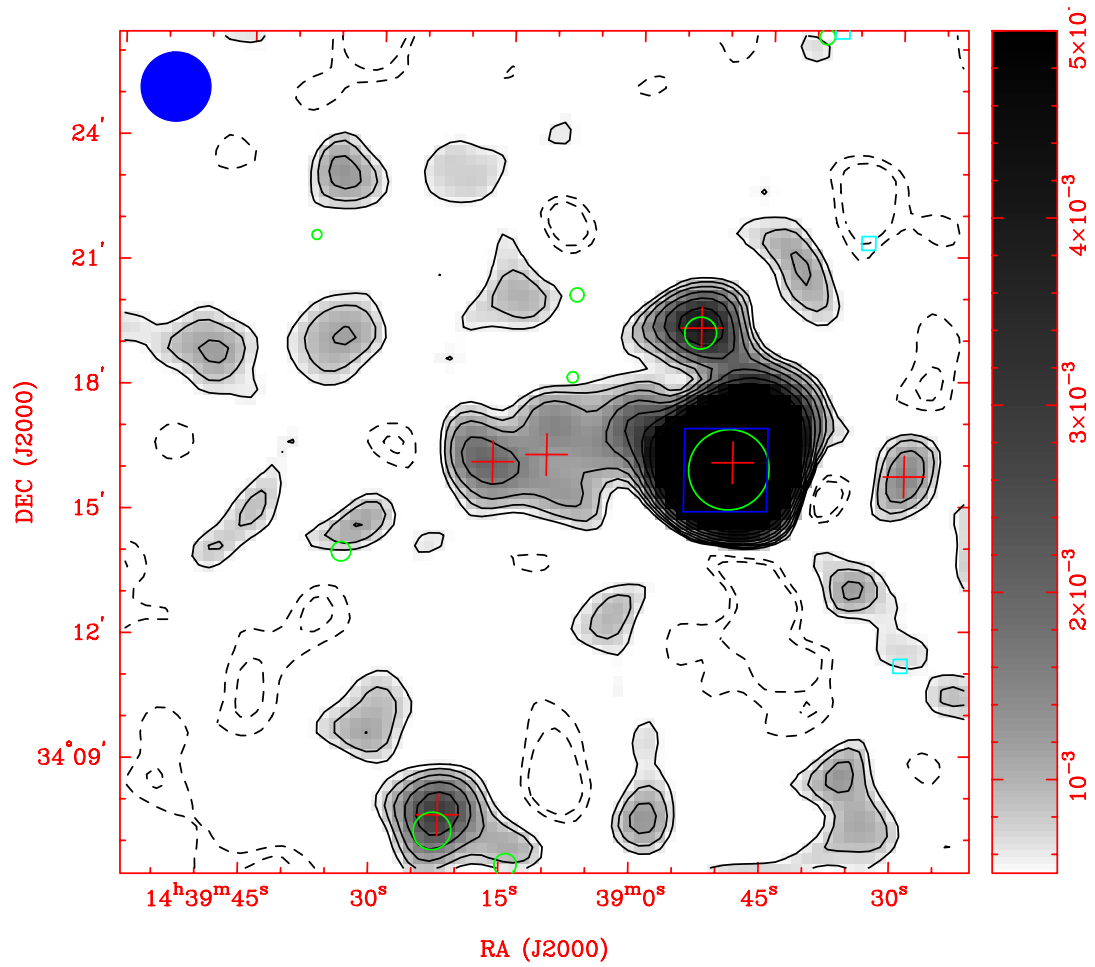

Figure 8. Extended PiGSS source not observed in NVSS. Contours are the same as in Figure 4.

(A color version of this figure is available in the online journal.)

times of those surveys. In the case of NVSS, that timescale is minutes.

As discussed above, we identify one source J143621+334120 that appears in PiGSS but in none of the other catalogs. With a flux density of $1.80 \pm 0.42 \mathrm{mJy}$, the source is a marginal detection at $4.3 \sigma$. For purely Gaussian image noise, the expectation over the entire image is of $\sim 0.1$ sources at this threshold or above. Thus, it is not unlikely that this is a random fluctuation or the result of increased noise threshold from systematic error. In a forthcoming paper we will investigate the variability of this source in daily and monthly images.

Since we do not have firm detection of any transient sources, we do not have a transient timescale. In this case, the snapshot rate $R$ (Equation (4)), which compares the number of transients in a two-epoch survey, is the appropriate statistic to make use of. We therefore set an upper limit to the transient rate for longduration transients, $R_{\text {month }} \lesssim 1 \mathrm{deg}^{-2}$ at $1 \mathrm{mJy}$ and $\lesssim 0.3 \mathrm{deg}^{-2}$ at $10 \mathrm{mJy}$. In the reverse case of identifying NVSS sources missing in PiGSS, we set a limit $R_{\text {minute }} \lesssim 0.3 \mathrm{deg}^{-2}$ for flux densities greater than $5 \mathrm{mJy}$. Exact limits for long-duration transients are plotted in Figure 12 along with rates from past observations.

Rates on transient and variable sources will improve significantly with the release of daily monitoring results for the Boötes field and yearly monitoring for the NGP PiGSS catalogs. For the former, we anticipate 2 orders of magnitude greater sensitivity to short-timescale phenomena at flux densities an order of magnitude higher. For the latter, we anticipate 3 orders of magnitude improvement in transient and variable rates.

The current set of limits for transients of duration 4 months places the most interesting limits on AGNs, ESEs, and RSNe, all of which can have durations of months to years. The case of III Zw 2 is instructive for extreme AGN variability; it exhibited a factor of $>20$ outburst rising from below $100 \mathrm{mJy}$ to greater than 1 Jy on a timescale of 1 year (Falcke et al. 1999; Brunthaler et al.
2005). III Zw 2 has exhibited episodic variability of this kind with a duty cycle of $\sim 20 \%$. Such a source would be readily detectable in this survey down to our detection threshold of $1 \mathrm{mJy}$. Thus, we can limit the number of III Zw 2 analog sources to be $\lesssim 0.2 \%$ of all radio sources. ESEs have timescales of months and amplitudes of order factor of 2 and therefore are harder to distinguish in a two-epoch comparison. Detailed analysis of daily light curves will be sensitive to ESE behavior.

\subsection{Flat-spectrum Sources}

Of particular interest is the construction of a flat-spectrum catalog from the PiGSS data. We identify 124 sources with $\alpha>-0.5$ and 39 sources with $\alpha>0$. If we restrict our sample to those within the half-power radius where fluxes are more accurate, then we find 26 sources with $\alpha>0$, or $8 \mathrm{deg}^{-2}$.

A single source from the LAT 1 year catalog falls in the Boötes field, 1FGL J1426.0+3403 (Abdo et al. 2010). This source is a probable match to the PiGSS source J142607+340433, which has a flux of $27.5 \pm 0.6 \mathrm{mJy}$. Matches to the other catalogs indicate a spectral index of $\sim-0.2$. The source is not identified in the CGRaBS catalog of flat-spectrum radio sources, presumably because it falls below the detection threshold for that catalog (Healey et al. 2008). The LAT catalog associates this source with the BL Lac object BZB J1426+3404 (Massaro et al. 2009). As the LAT catalog becomes more sensitive and detects more transient and variable sources, we can expect an increased number of LAT sources to fall into these fields. The next PiGSS paper will study short-term variability of sources in the field, which may be a stronger predictor of gamma-ray activity than spectral index.

The BZ catalog identifies three blazars, BL Lac objects, and radio loud quasars in the Boötes field, using $1.4 \mathrm{GHz}$ radio flux data and optical spectra (Massaro et al. 2009). The BZ catalog has an average density of $\sim 0.1$ sources $\mathrm{deg}^{-2}$. The higher 

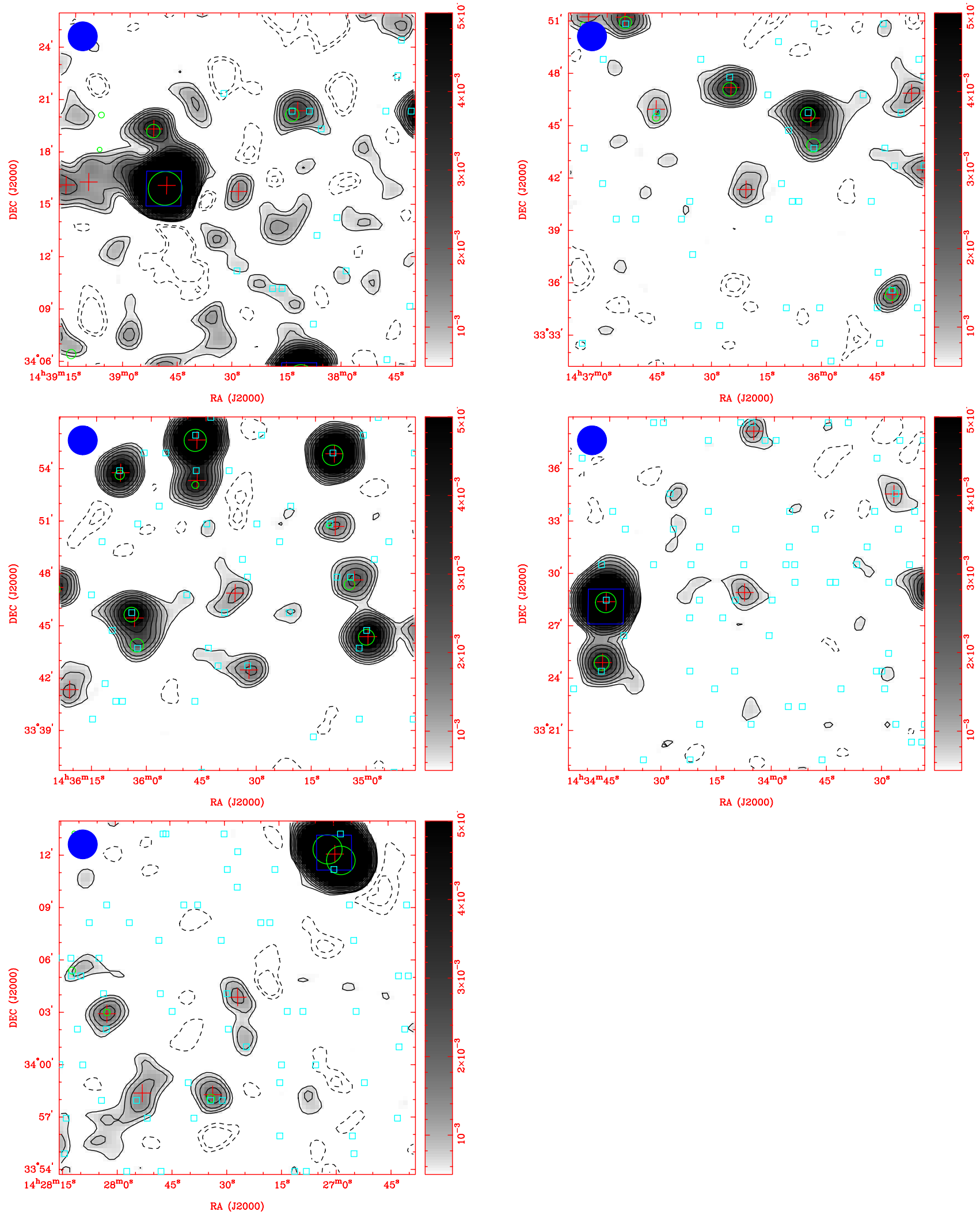

Figure 9. Images of unresolved PiGSS sources not identified in NVSS and WSRT-1400 and within the half-power contour of the image. The source without an identification is located at the center of each image.

(A color version of this figure is available in the online journal.)

number density in the Boötes field is due to the non-uniformity of the available optical spectroscopy. The $\sim 10^{2}$ flat-spectrum sources identified via PiGSS spectral indices indicate an order of magnitude increase in the number of blazar candidates. Over the $10^{4} \mathrm{deg}^{2}$ of PiGSS, we expect to identify $\sim 10^{4}$ flat-spectrum sources above a flux density of $10 \mathrm{mJy}$. This is 


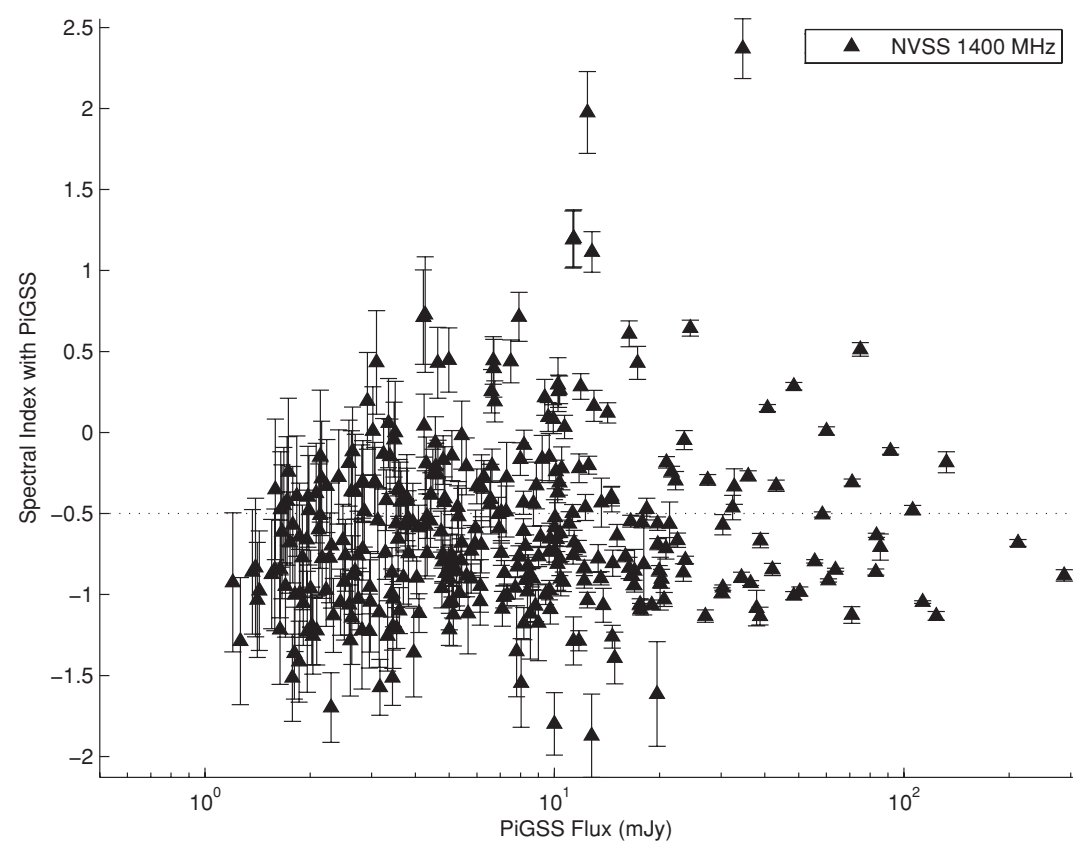

Figure 10. Spectral indices generated from PiGSS with respect to the NVSS. The dashed line indicates the cutoff of $\alpha>-0.5$ for flat-spectrum sources.

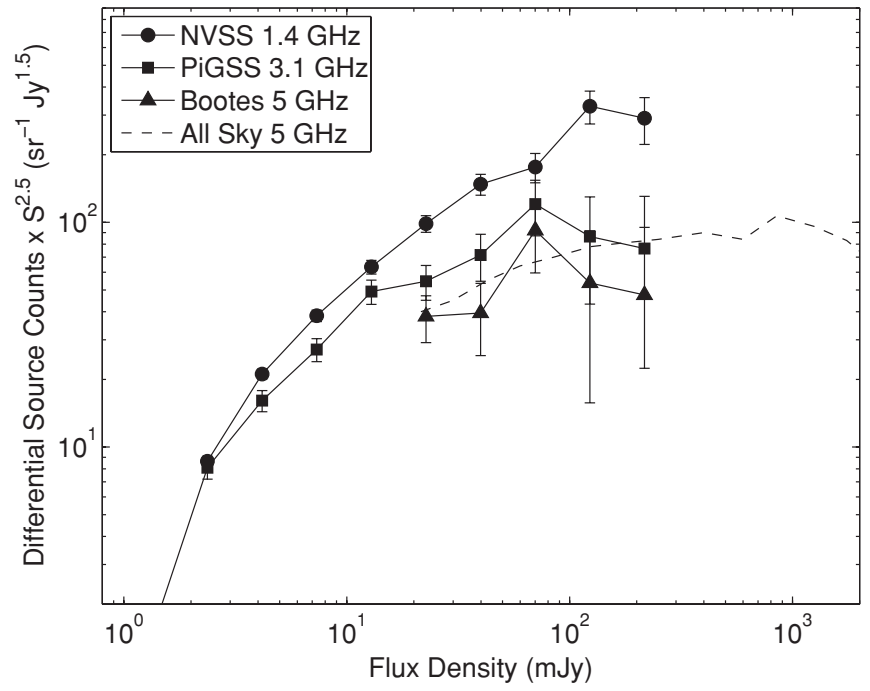

Figure 11. Number counts as a function of flux density from PiGSS data (squares), NVSS sources in the same field (filled circles), GB6 sources in the same field (filled triangles), and $5 \mathrm{GHz}$ source counts from the full GB6 catalog (dashed line; Gregory et al. 1996).

an order of magnitude increase over the 1625 flat-spectrum sources identified in CGRaBS. Comparison of PiGSS sources in Boötes with the extensive NDWFS imaging and spectroscopic results should provide a compelling characterization of the flatspectrum population in this field.

\section{CONCLUSIONS}

We present the first results from the PiGSS in this paper. This includes a description of the overall survey strategy and goals with an emphasis on transient and variable source characterization. The data presented in this paper represent less than $3 \%$ of the total data that will be obtained in PiGSS. These data are a deep image of a region that is approximately $10 \mathrm{deg}^{2}$ in the Boötes field. Comparison of the PiGSS results with existing surveys of the field, especially NVSS, provides an important

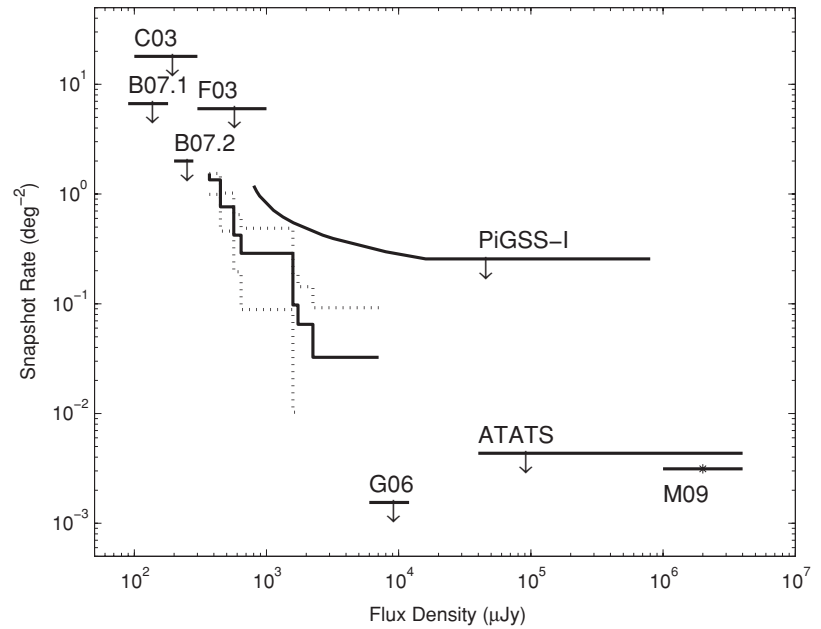

Figure 12. Two-epoch transient rates from PiGSS and other surveys as a function of flux density. The curved solid black line labeled PiGSS-I is the limit from this paper. The solid black line with step functions shows the rate from Bower et al. (2007) while the dotted lines show the $2 \sigma$ upper and lower bounds. The arrows show $2 \sigma$ upper limits for transients from Bower et al. (2007) with a 1 year timescale (B07.1), 2 month timescale (B07.2), and for transients from the comparison of the $1.4 \mathrm{GHz}$ NVSS and FIRST surveys (G06; Gal-Yam et al. 2006), from the Carilli et al. (2003) survey (labeled C2003), from the Frail et al. (2003) survey (labeled F2003), from ATATS (Croft et al. 2010), and from the Matsumura et al. (2009) survey (labeled M09).

verification of the fidelity of the PiGSS data. We detect $\sim 10^{2}$ flat-spectrum sources in this early stage of PiGSS. We identify one PiGSS source that does not appear in any other radio catalog. This source is possibly transient or strongly variable but may also be due to fluctuations in the noise; a future paper will examine the daily and monthly images of this source. We set upper limits on transients with lifetimes of minutes and months. The complete PiGSS catalog will provide a significant exploration of transient parameter space on timescales that range from minutes to years.

The authors acknowledge the generous support of the Paul G. Allen Family Foundation, which has provided major support for 
design, construction, and operations of the ATA. Contributions from Nathan Myhrvold, Xilinx Corporation, Sun Microsystems, and other private donors have been instrumental in supporting the ATA. The ATA has been supported by contributions from the US Naval Observatory in addition to National Science Foundation grants AST-050690, AST-0838268, and AST-0909245. We dedicate this paper to the memory of the late Don Backer: mentor, colleague, and friend.

\section{REFERENCES}

Abazajian, K., et al. 2003, AJ, 126, 2081

Abdo, A. A., et al. 2010, ApJS, 188, 405

Ashby, M. L. N., et al. 2009, ApJ, 701, 428

Baars, J. W. M., Genzel, R., Pauliny-Toth, I. I. K., \& Witzel, A. 1977, A\&A, 61,99

Becker, R. H., Helfand, D. J., White, R. L., \& Proctor, D. D. 2010, AJ, 140, 157

Becker, R. H., White, R. L., \& Helfand, D. J. 1995, ApJ, 450, 559

Berger, E., et al. 2001, Nature, 410, 338

Bower, G. C., Roberts, D. A., Yusef-Zadeh, F., Backer, D. C., Cotton, W. D., Goss, W. M., Lang, C. C., \& Lithwick, Y. 2005, ApJ, 633, 218

Bower, G. C., Saul, D., Bloom, J. S., Bolatto, A., Filippenko, A. V., Foley, R. J., \& Perley, D. 2007, ApJ, 666, 346

Brunthaler, A., Falcke, H., Bower, G. C., Aller, M. F., Aller, H. D., \& Teräsranta, H. 2005, A\&A, 435, 497

Brunthaler, A., Menten, K. M., Reid, M. J., Henkel, C., Bower, G. C., \& Falcke, H. 2009, A\&A, 499, L17

Calabretta, M. R., \& Greisen, E. W. 2002, A\&A, 395, 1077

Carilli, C. L., Ivison, R. J., \& Frail, D. A. 2003, ApJ, 590, 192

Condon, J. J. 1974, ApJ, 188, 279

Condon, J. J., Cotton, W. D., Greisen, E. W., Yin, Q. F., Perley, R. A., Taylor, G. B., \& Broderick, J. J. 1998, AJ, 115, 1693

Croft, S., et al. 2010, ApJ, 719, 45

de Vries, W. H., Morganti, R., Röttgering, H. J. A., Vermeulen, R., van Breugel, W., Rengelink, R., \& Jarvis, M. J. 2002, AJ, 123, 1784

de Zotti, G., Massardi, M., Negrello, M., \& Wall, J. 2010, A\&AR, 18, 1

Falcke, H., et al. 1999, ApJ, 514, L17

Fassnacht, C. D., Xanthopoulos, E., Koopmans, L. V. E., \& Rusin, D. 2002, ApJ, 581,823

Fiedler, R. L., et al. 1987, ApJS, 65, 319

Frail, D. A., Kulkarni, S. R., Berger, E., \& Wieringa, M. H. 2003, AJ, 125, 2299

Gal-Yam, A., et al. 2006, ApJ, 639, 331

Gehrels, N. 1986, ApJ, 303, 336

Gregory, P. C., Scott, W. K., Douglas, K., \& Condon, J. J. 1996, ApJS, 103, 427

Healey, S. E., et al. 2008, ApJS, 175, 97

Hessels, J. W. T., Stappers, B. W., van Leeuwen, J., \& the LOFAR Team 2009, in ASP Conf. Ser. 407, The Low-Frequency Radio Universe, ed. D. J. Saikia, D. A. Green, Y. Gupta, \& T. Venturi (San Francisco, CA: ASP), 318
Hewish, A., Bell, S. J., Pilkington, J. D. H., Scott, P. F., \& Collins, R. A. 1969, Nature, 224, 472

Hopkins, A. M., Miller, C. J., Connolly, A. J., Genovese, C., Nichol, R. C., \& Wasserman, L. 2002, AJ, 123, 1086

Hughes, P. A., Aller, H. D., \& Aller, M. F. 1992, ApJ, 396, 469

Hull, C., Bower, G., \& Croft, S. 2010, PASP, in press (arXiv:1010.1064)

Hyman, S. D., Lazio, T. J. W., Kassim, N. E., \& Bartleson, A. L. 2002, AJ, 123 1497

Hyman, S. D., Lazio, T. J. W., Kassim, N. E., Ray, P. S., Markwardt, C. B., \& Yusef-Zadeh, F. 2005, Nature, 434, 50

Hyman, S. D., Wijnands, R., Lazio, T. J. W., Pal, S., Starling, R., Kassim, N. E., \& Ray, P. S. 2009, ApJ, 696, 280

Ivezić, Ž., et al. 2002, AJ, 124, 2364

Jannuzi, B. T., et al. 2004, BAAS, 36, 1478

Keating, G., Barott, J., \& Allen Telescope Array Team 2009, in American Astronomical Society Meeting Abstracts, Vol., 214, 601.06

Law, C., Gaensier, B., \& Bower, G. 2010, ApJ, submitted

Law, N. M., et al. 2009, PASP, 121, 1395

Levinson, A., Ofek, E. O., Waxman, E., \& Gal-Yam, A. 2002, ApJ, 576, 923

Macquart, J., et al. 2010, PASA, 27, 272

Massaro, E., Giommi, P., Leto, C., Marchegiani, P., Maselli, A., Perri, M., Piranomonte, S., \& Sclavi, S. 2009, A\&A, 495, 691

Matsumura, N., et al. 2009, AJ, 138, 787

McLaughlin, M., et al. 2006, Nature, 439, 817

Murphy, T., et al. 2010, MNRAS, 402, 2403

Myers, S. T., et al. 2003, MNRAS, 341, 1

Ofek, E. O., Breslauer, B., Gal-Yam, A., Frail, D., Kasliwal, M. M., Kulkarni, S. R., \& Waxman, E. 2010, ApJ, 711, 517

Osten, R. A., Hawley, S. L., Allred, J., Johns-Krull, C. M., Brown, A., \& Harper, G. M. 2006, ApJ, 647, 1349

Rau, A., et al. 2009, PASP, 121, 1334

Sault, R. J., Staveley-Smith, L., \& Brouw, W. N. 1996, A\&AS, 120, 375

Sault, R. J., Teuben, P. J., \& Wright, M. C. H. 1995, in ASP Conf. Ser. 77, Astronomical Data Analysis Software and Systems IV, ed. R. A. Shaw, H. E. Payne, \& J. J. E. Hayes (San Francisco, CA: ASP), 433

Schilizzi, R., et al. 2007, http://www.skatelescope.org/PDF/memos/100_Memo Schilizzi.pdf

Senkbeil, C. E., Ellingsen, S. P., Lovell, J. E. J., Macquart, J., Cimò, G., \& Jauncey, D. L. 2008, ApJ, 672, L95

Sesar, B., et al. 2007, AJ, 134, 2236

Siemion, A., et al. 2010, Acta Astron., 67, 1342

Stanghellini, C., O’Dea, C. P., Dallacasa, D., Baum, S. A., Fanti, R., \& Fanti, C. 1998, A\&AS, 131, 303

Voges, W., et al. 1999, A\&A, 349, 389

Waldram, E. M., Pooley, G. G., Davies, M. L., Grainge, K. J. B., \& Scott, P. F. 2010, MNRAS, 404, 1005

Weiler, K. W., Panagia, N., Montes, M. J., \& Sramek, R. A. 2002, ARA\&A, 40, 387

Welch, J., et al. 2009, in Advances in Radio Telescopes, ed. J. Baars, R Thompson, \& L. D' Addario, Proc. IEEE, 97, 1438

Williams, P. K. G., \& Bower, G. C. 2010, ApJ, 710, 1462 